\title{
Thermal conductivity of unidirectional composites consisting of randomly dispersed glass fibers and temperature-dependent polyethylene matrix
}

https://doi.org/10.1515/secm-2019-0024

Received April 8, 2019; accepted July 12, 2019

\begin{abstract}
This extensive study investigated the influence of microstructure on the effective transverse thermal conductivity of unidirectional glass fiber reinforced composites, in which the fibers are randomly dispersed and the thermal conductivity of polyethylene matrix is a function of test temperature. The microstructure is characterized by parameters such as the number of fibers, fiber volume fraction, fiber size, fiber arrangement and thermal property contrast. Firstly, a simple algorithm is developed to automatically generate closest-to-real random array of fibers in unit cell to reconstruct the composite microstructure. Then, the established two-dimensional random twocomponent composite unit cell is solved using finite element simulation and the obtained effective thermal conductivities are compared with the theoretical predictions and the experimental results. Subsequently, the effects of microstructure parameters and test temperature are investigated, respectively. It is found that the finite element predicted properties are in very good agreement with the experimental predictions, while they are always lower than the analytically predicted properties. These results can find applications in the design of composite materials taking into account the fiber distribution morphology.
\end{abstract}

Keywords: Fiber reinforced composites; Microstructure; Randomness; Thermal conductivity; Finite element method

\section{Introduction}

The incorporation of fibers, i.e. carbon fibers, glass fibers, boron fibers, and microparticles/nanoparticles in matrix

\footnotetext{
Wan-Qing Lin, Yu-Xuan Zhang: College of Civil Engineering and Architecture, Henan University of Technology, Zhengzhou 450001, China

*Corresponding Author: Hui Wang: College of Civil Engineering and Architecture, Henan University of Technology, Zhengzhou 450001, China, E-mail: huiwang@haut.edu.cn
}

material is facilitated to form various composites [1-6]. The effective thermal conductivity of them is one of the most important quantities characterizing energy transport in a vast range of engineering applications [7-12]. Generally, fibers are assumed to be regularly dispersed in matrix in square or hexagonal mode for simplicity so that a relative simple composite model can be obtained for thermal analysis [13-17]. Actually, the fiber distribution morphology is very complex in matrix. They are generally dispersed in matrix in random arrangement [18-23]. Thus, there is an increasing demand to establish proper random composite model for applications so that the fiber distribution may match the realistic arrangement in composite as possible [24-26]. Except for digital image technique, which strongly requires special device and software, to obtain the real microstructure $[27,28]$, some attempts have been made by developing random algorithm to generate closest-to-real random composite models for investigating the effect of fiber distribution mode on the effective thermal conductivity of composites. For example, Wang et al. studied the influence of clustered natural fibers regularly or randomly distributed in cementitious material on the effective thermal conductivity of composites [29]. Fang et al. investigated the geometrical effect of fibers in the unidirectional fiber-reinforced polymer composite [30]. Yang et al. developed a random sequential expansion algorithm for generating random fiber distributions in the cross-sectional plane of unidirectional fiber-reinforced composite [31]. Montesano and his partners developed an algorithm based on event-driven molecular dynamics theory to rapidly generate periodic representative volume elements with uniform or nonuniform filler distributions for both unidirectional fiber-reinforced and spherical particle-reinforced composites [3, 32]. Besides, an algorithm based on constrained optimization formulation was developed by Pathan et al. to generate random distributions of cylindrical fibers and spherical particles [33]. However, the existing algorithms look mathematically complex, moreover, the temperature dependence of thermal conductivity of matrix material $[34,35]$ was seldom considered for investigating the effective thermal conductivities of fiber-reinforced composites. 
This work aims to the prediction of transverse effective thermal conductivity of unidirectional composite materials containing randomly dispersed long fibers by finite element simulation. The random pattern of fibers is generated by a simple random algorithm. The thermal conductivity of matrix material is assumed to be dependent of its test temperature. With given fiber size and number of fibers, the present algorithm really randomly generates the positions of circular fibers for a specific volume fraction in a square matrix region by requiring that the fibers don't intersect. Such condition can be ensured by making the distances between the centers of the new fiber and the existing fibers that have been created greater than the summation of their radius. This automated generation process will not stop until the specific fiber volume fraction reaches. As there are no limitations of fiber size, material, and shape in the generation process, it is considerably easy to generate a combination of randomly distributed fibers with two or more different materials, sizes or shapes. Subsequently, the influences of fiber arrangement, fiber size, thermal conductivity ratio between components, and fiber volume fraction are taken into account in numerical simulations under different test temperatures. The resulted predictions are analyzed and compared with those from various analytical models [11] and experiments [34].

The paper is organized as follows. In Section 2, the algorithm for the generation of computational unit cell modelling containing randomly distributed fibers is depicted. Then, in Section 3, the finite element simulation is performed for the calculation of transverse effective properties of unidirectional fiber reinforced composites. In Section 4, numerical results are discussed and compared with analytical models and experiments. Finally, the work is summarized in Section 5.

\section{Generation of random fiber arrangement}

In this section, random fiber arrangement in unit cell is automatically generated by a simple algorithm, which is designed based on the existing literature [19]. For a random unit cell model with uniform fiber size distribution, it is assumed that the fibers are randomly distributed in it and don't intersect with each other or the cell boundaries. This restriction is helpful to ease the determination of fiber volume fraction and more importantly, avoid mesh distortion in finite element simulation.

Firstly, the pre-defined geometric parameters of the unit cell include the number of fibers, fiber radius and the length of unit cell. The relationship between the geometric parameters and the fiber volume fraction can be expressed as

$$
v_{f}=\frac{n \times \pi r_{f}^{2}}{a^{2}}
$$

where $v_{f}, n$ and $a$ are the fiber volume fraction, the number of fibers with radius $r_{f}$, and the side length of square unit cell, respectively. It appears that when the number of fibers, the fiber size, and the fiber volume fraction are given, the size of unit cell can be evaluated by

$$
a=\sqrt{\frac{n \times \pi r_{f}^{2}}{v_{f}}}=r_{f} \sqrt{\frac{n \times \pi}{v_{f}}}
$$

from which it is found that the cell size is obviously a function of the fiber volume fraction, the number of fibers, and the fiber size. In this study, it is assumed that the fiber size is identical and specified, and therefore, the fiber volume fraction and the number of fibers are two important morphological parameters to be studied in this work. Next, a simple algorithm following the procedure below is presented to generate the random arrangement of fibers in the unit cell, which is illustrated in Fig. [1]. s
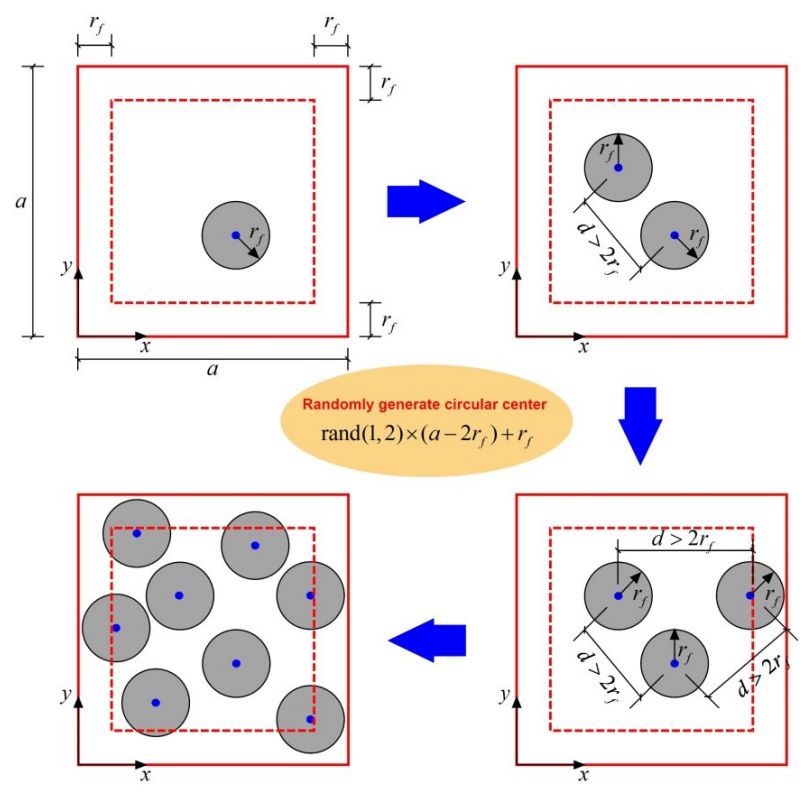

Figure 1: Schematic illustration of the procedure of the present algorithm

Step 1: The unit cell with side length $a$ is considered with the origin of coordinates at the left and low corner of it. In order to avoid the fiber intersecting with the cell boundaries, a reduced square region with side length $a-2 r_{f}$ is defined (see the dashed square region in Fig. 1). Then, a 
random point standing for the center of the first fiber with coordinate $\left(x_{1}, y_{1}\right)$ is firstly created by

$$
\left(x_{1}, y_{1}\right)=\operatorname{rand}(1,2) \times\left(a-2 r_{f}\right)+r_{f}
$$

where $\operatorname{rand}(1,2)$ is the inbuilt function of MATLAB to simultaneously produce two random numbers in the region $(0,1)$.

Step 2: A new random point $\left(x_{\text {new }}, y_{\text {new }}\right)$ representing the center of a new fiber is created similarly in the reduced region by

$$
\left(x_{\text {new }}, y_{\text {new }}\right)=\operatorname{rand}(1,2) \times\left(a-2 r_{f}\right)+r_{f}
$$

If the distance $d$ between the new fiber and the first fiber is larger than $2 r_{f}$, the newly generated point is accepted and reset as the position of the second fiber. If not, the new fiber is discarded and you have to repeat such procedure until the second point is created successfully.

Step 3: Repeat Step 2 to generate the specified number of fibers in the unit cell and ensure that the distances between the new fiber and other existing fibers are all greater than $2 r_{f}$.

From the procedure above, it is seen that the algorithm is very simple, and doesn't require too much mathematical foundation. To investigate the applicability of the algorithm for various numbers of fibers, it is assumed that there are 50, 100, 200 and 400 fibers randomly dispersed in the unit cells, respectively. The fiber volume fraction $v_{f}$ is set to be $20 \%$ and the fiber radius $r_{f}$ is $7 \mu \mathrm{m}$, which corresponds to the glass fiber considered in this study [34]. Fig. 2 shows the resulted random geometric models and it is found that the present algorithm can effectively generate random distributions of fibers in the unit cells under the given value of fiber volume fraction. Besides, to illustrate the applicability of the algorithm for higher fiber volume fraction, i.e. $50 \%$ or higher, which is a common value in the real composite material, Fig. 3 depicts examples of generated distributions with $v_{f}=54 \%$, and it is seen that the fiber distributions show excellent randomness. Additionally, because of the randomness of fiber position, the algorithm can produce different fiber distributions even if the geometric parameters including the fiber volume fraction, the fiber size, and the fiber quantity are unchanged. Fig. 5 shows the examples of different random models generated by the present algorithm with $v_{f}=20 \%$ and $n_{f}=100$. Therefore, all examples demonstrate the full applicability of the present algorithm to generate really random microstructures of composites over a large range of fiber volume fractions and fiber quantities. In addition, it is necessary to point out that higher fiber volume fraction can be achieved by following the steps above and introducing polar coordinates around each existing circle. As a result, the fiber volume fraction can be up to $65 \%$. Fig. 4 display examples of generated random distributions with $v_{f}=65 \%$ for different fiber numbers.
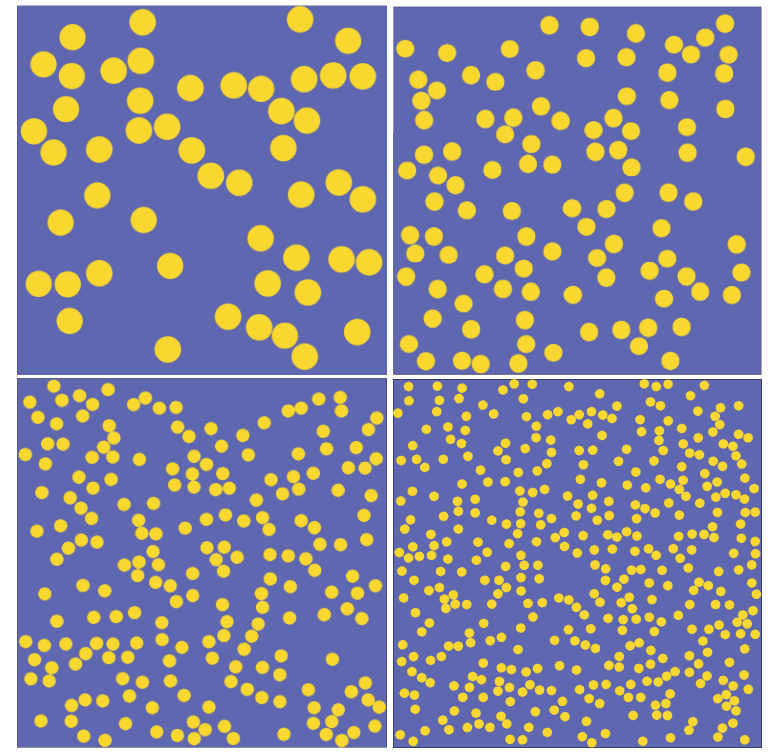

Figure 2: The generated random models with 50, 100, 200 and 400 fibers for $v_{\mathrm{f}}=20 \%$

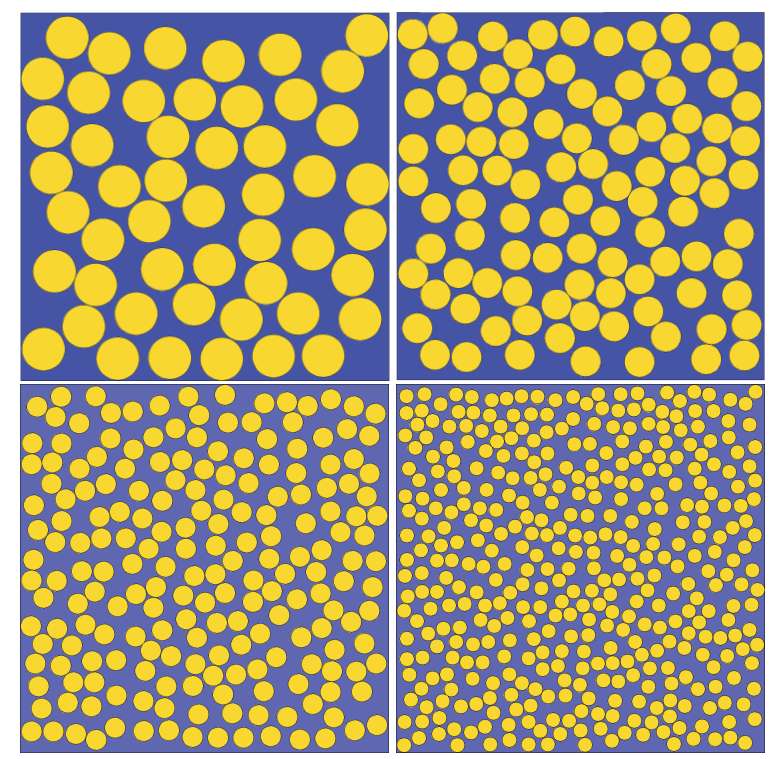

Figure 3: The generated random models with 50, 100, 200 and 400 fibers for $v_{\mathrm{f}}=54 \%$ 


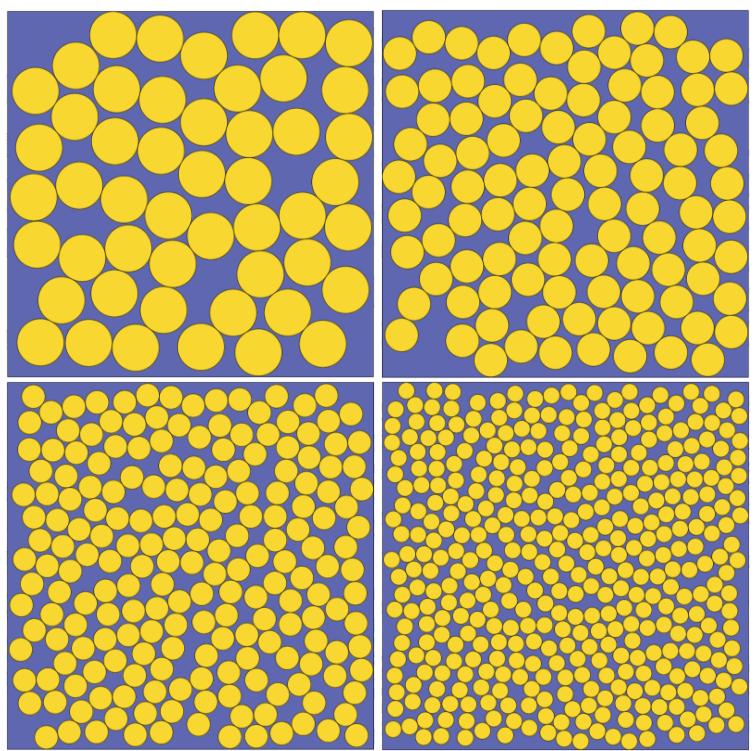

Figure 4: The generated random models with 50, 100, 200 and 400 fibers for $v_{\mathrm{f}}=65 \%$
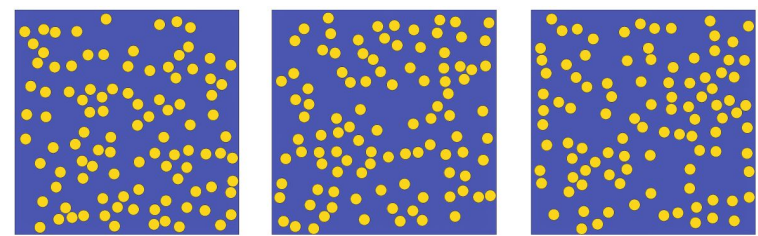

Figure 5: Three different random arrangements of fibers generated by the present algorithm with $v_{f}=20 \%$ and $n_{f}=100$

\section{Numerical simulation}

Once the random microstructures of composites are generated, we can perform the prediction of effective transverse thermal property of composites from the properties of their constituents, and the given filler volume fraction. In this section, the finite element method is used to achieve such objective and also validate the present algorithm. The 3node plane linear elements are used to discretize all composite microstructure models. For comparison, the regular geometric models including the square and hexagonal fiber arrangements are established, as shown in Fig. 6. Moreover, the existing experimental results of composite consisting of the glass fiber and the polyethylene (HDPE) matrix material [34] is considered to verify the computational models. Both the glass fiber and the matrix are considered to be isotropic and homogeneous. The glass fiber with diameter $d_{f}=14 \mu \mathrm{m}$ has constant thermal conductivity $k_{f}=1.03 \mathrm{~W} /(\mathrm{mK})$, while the thermal conductivity $k_{m}$ of matrix material changes with respect to the test temperature, as shown in Fig. 7.

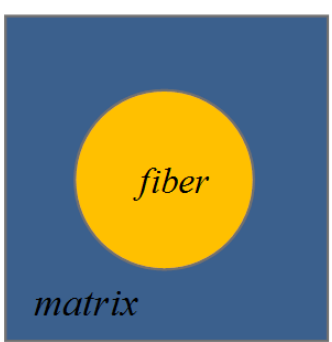

(a)

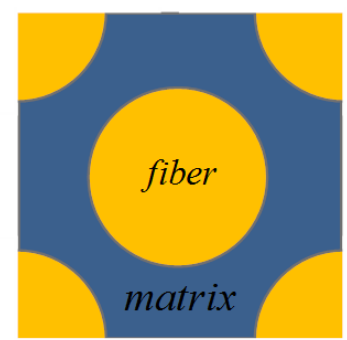

(b)
Figure 6: Unit cells with regular (a) square and (b) hexagonal fiber patterns

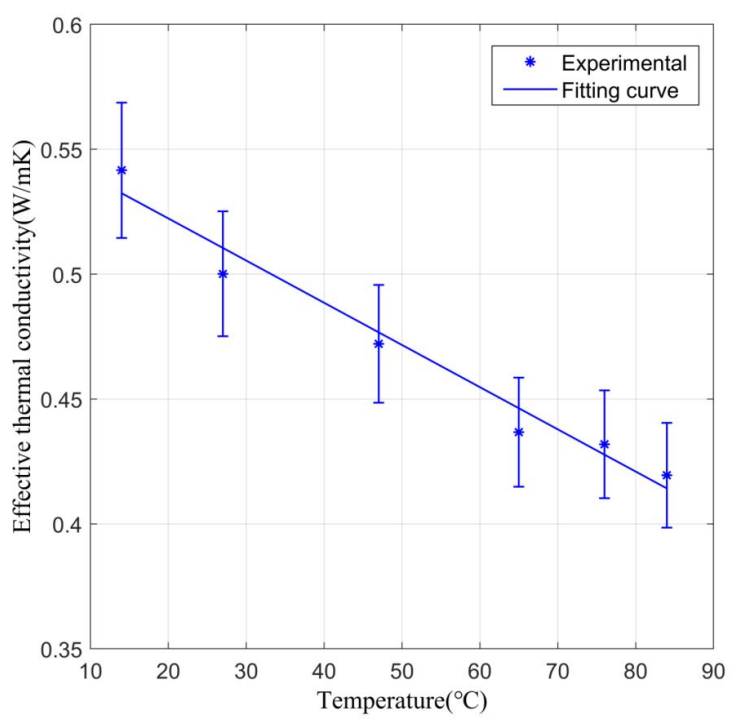

Figure 7: The thermal conductivity of HDPE matrix varies with test temperature

\subsection{Finite element model}

Fig. 8 shows a numerical model and boundary conditions used for finite element simulation, in which the steadystate heat transfer in the $x y$ plane is governed by the following Laplace equations

$$
\nabla^{2} T_{m}=0, \quad \nabla^{2} T_{f}=0
$$

where $T_{m}$ and $T_{f}$ are temperature distributions in the matrix and fiber material phases, respectively. 


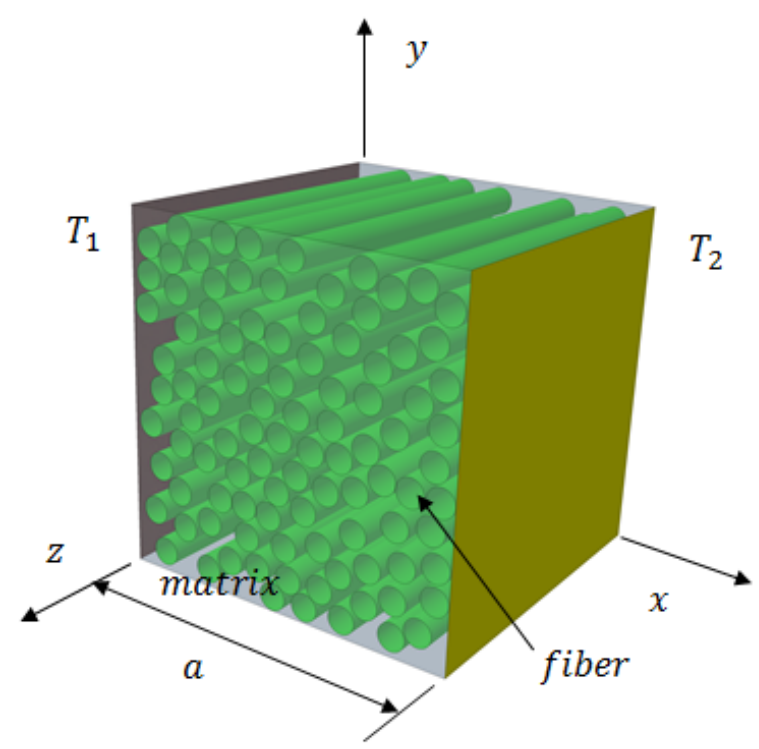

Figure 8: Schematic diagram of transverse heat transfer in the unit cell

In simulation, the upper and lower boundaries of the unit cell are assumed as thermal insulation boundaries and the left and right boundaries are regarded as temperature boundaries. The related boundary conditions can be written as

$$
\left.k_{m} \frac{\partial T}{\partial y}\right|_{y=0, y=a}=0,\left.\quad T\right|_{x=0}=T_{1},\left.\quad T\right|_{x=a}=T_{2}
$$

Moreover, the effect of thermal contact resistance on the interface of fiber and matrix is neglected, so the interfacial continuum is written as

$$
T_{f}=T_{m}, \quad k_{f} \frac{\partial T_{f}}{\partial n}=k_{m} \frac{\partial T_{m}}{\partial n}
$$

where $n$ is the unit normal to the fiber/matrix interface.

Due to the randomness of fiber distribution, the composite is assumed to be orthotropic on the cross-section plane. Thus, based on Fourier's law, the heat flux components $q_{x}$ and $q_{y}$ in the computational domain can be expressed by the temperature gradient [36]

$$
\left[\begin{array}{l}
q_{x} \\
q_{y}
\end{array}\right]=-\left[\begin{array}{ll}
k_{x} & \\
& k_{y}
\end{array}\right]\left[\begin{array}{l}
\frac{\partial T}{\partial x} \\
\frac{\partial T}{\partial y}
\end{array}\right]
$$

from which the effective thermal conductivity of the composite along the $x$-direction can be approximated by [29, 34, 37]

$$
k_{x} \approx \frac{\bar{q}_{x} a}{\Delta T} \approx \frac{\bar{q}_{x} a}{T_{1}-T_{2}}
$$

in which $\bar{q}_{x}$ is the average heat flux component on the surface perpendicular to the $x$ axis, i.e. the surface $x=a$ with the constant temperature constraint $T_{2}$. In this study, the left and right boundary temperatures are $T_{1}=400 \mathrm{~K}$ and $T_{2}=300 \mathrm{~K}$, respectively.

\subsection{Analysis of numerical convergence}

The governing equations (5) with the boundary conditions (6) and the perfect interfacial condition (7) are solved using finite elements. Here, the thermal conductivity of HDPE matrix material is taken as $0.4367 \mathrm{~W} /(\mathrm{mK})$, which corresponds to the test temperature $65^{\circ} \mathrm{C}$ [34]. To obtain converged solutions, the numerical convergence criterion is defined such that the maximum relative difference in the predicted effective thermal conductivity is less than $0.1 \%$ when increasing the number of elements from the current meshing scheme to a new one. Fig. 9 shows the meshing schemes for the two microstructures including 50 and 400 fibers, respectively, with 50\% fiber volume fraction. It is found that the converged results can be achieved with about 11000 and 60000 elements for the cases of 50 and 400 fibers, respectively. Also, it is clear that the number of finite elements needed to obtain a converged result strongly depends on the size of computational cell and the number of fibers. Moreover, Fig. 10 displays example of the local distributions of temperature and heat flux component $q_{x}$ in the unit cell containing 400 randomly dispersed fibers for the cases of $v_{f}=14 \%$ and $27 \%$. From this figure, it appears that the temperature distributions look almost same for the two different fiber volume fractions, while the local heat flux density is dramatically influenced by the filler distribution and filler volume concentration, and the maximum heat flux for $v_{f}=14 \%$ is greatly less than that for $v_{f}=27 \%$. Besides, the heat flux around the fibers is much higher than that in the matrix. This can be attributed to the higher thermal conductivity of fiber.

\section{Results and discussion}

In this study, the influences of test temperature, fiber volume fraction, fiber arrangement and thermal property contrast are studied for the composite unit cell models. Moreover, in order to well understand the applicability of random model, the existing theoretical Gurtman model [11], which is based on the periodic permutation composite material, is given as

$$
k_{x}=v_{f} k_{f}\left(1+v_{m} A\right)+v_{m} k_{m}\left(1-v_{f} A\right)
$$



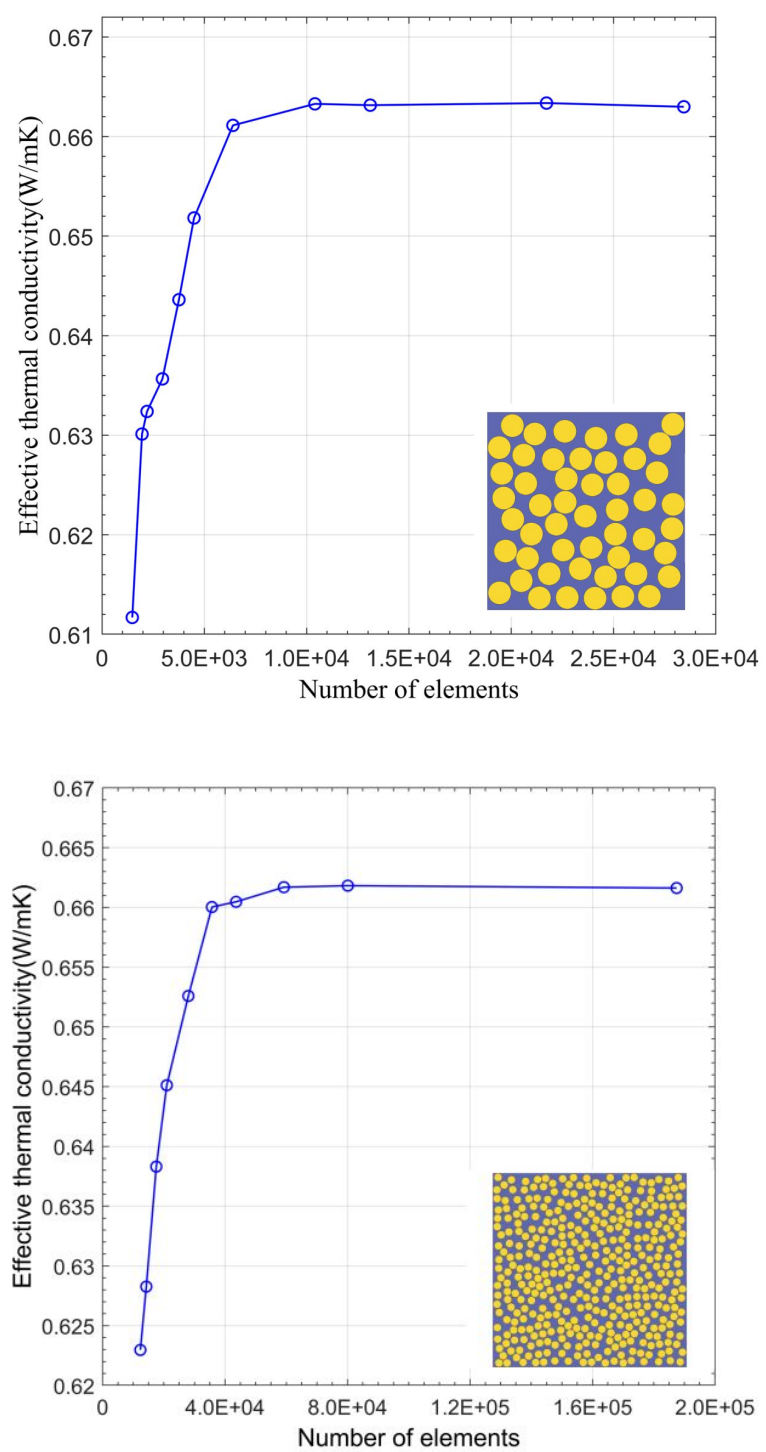

Figure 9: Convergence study for different mesh refinement levels for the composite microstructures with 50 and 400 fibers

with

$$
A=\frac{k_{m}-k_{f}}{\left(k_{m}+k_{f}\right)\left(1+v_{f}\right)}
$$

where $v_{m}=1-v_{f}$ is the volume fraction of matrix.

\subsection{The influence of random distribution}

Due to the real randomness of each position of fiber, the fiber distribution generated every time doesn't keep same even with same geometric parameter input, as shown in Fig. 5. Thus, investigating the influence of different random distributions of fibers on the effective thermal con- (a)

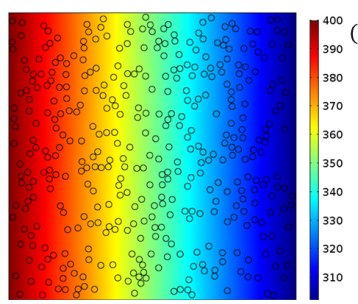

(c)
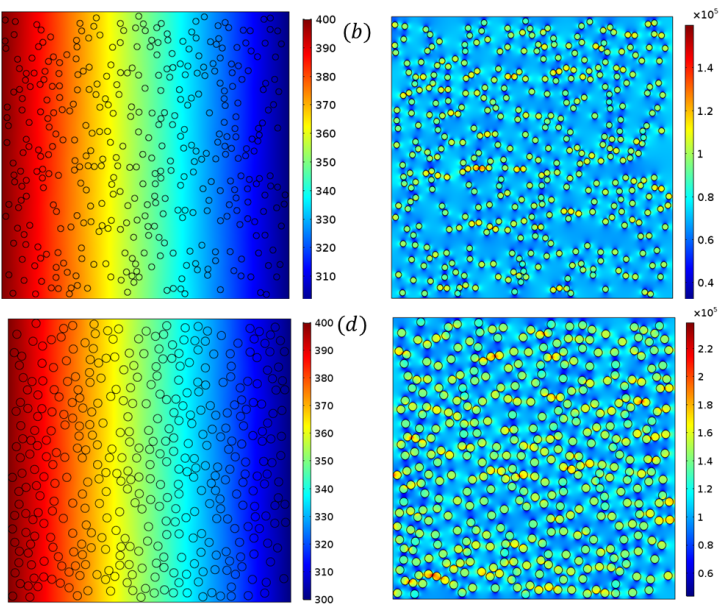

Figure 10: Distributions of temperature and heat flux component along the $x$-direction in the unit cells with 400 randomly dispersed fibers for $v_{f}=14 \%$ and $27 \%$

ductivity of composites is of great importance. Tables 1-3 list numerical results of three different fiber arrangements related to the given number of fibers and fiber volume fractions, respectively. Here, the fiber volume fraction covers a large range from low (14\%) to high (54\%) values and the number of fibers in unit cell changes from 50 to 600 . It is indicated from Tables 1-3 that the effective thermal conductivity $k_{x}$ is approximately independent of the fiber packing arrangement for each volume fraction. Therefore, only one fiber random packing arrangement is considered in the remainder of this study. Moreover, it is observed that the number of fibers randomly dispersed in the unit cell has slight influence on the effective thermal conductivity of composite.

\subsection{The influence of test temperature}

In this section, the effective thermal conductivity of composites in different test temperatures ranging from $8^{\circ} \mathrm{C}$ to $85^{\circ} \mathrm{C}$ is investigated. The fiber volume fraction is taken as $14 \%$ and $27 \%$, respectively, for the sake of comparison to the experimental results. Figs. 11 and 12 present the variations of the effective thermal conductivity $k_{x}$ as a function of test temperature for composites comprised of 50, 100, 200 and 400 randomly dispersed fibers, respectively. Correspondingly, the predictions from the regular square and hexagonal models, the experiment [34] and the theoretical Gurtman model [11] are also provided in Figs. 11 and 12 for comparison, in which the error bar has been included to represent the estimated uncertainty in the thermal conductivity data of $\pm 5 \%$. It is found that all predictions de- 
Table 1: Influence of random distributions of fibers on the effective thermal conductivity of composites with $v_{f}=14 \%$

\begin{tabular}{lccccc}
\hline & \multicolumn{5}{c}{ Number of fibers } \\
& 50 & 100 & 200 & 400 & 600 \\
\hline Random 1 & 0.4983 & 0.4943 & 0.4991 & 0.4980 & 0.4990 \\
Random 2 & 0.5000 & 0.4990 & 0.4996 & 0.4991 & 0.4991 \\
Random 3 & 0.4989 & 0.4991 & 0.4993 & 0.4989 & 0.4992 \\
\hline
\end{tabular}

Table 2: Influence of random distributions of fibers on the effective thermal conductivity of composites with $v_{f}=27 \%$

\begin{tabular}{lccccc}
\hline & \multicolumn{5}{c}{ Number of fibers } \\
& 50 & 100 & 200 & 400 & 600 \\
\hline Random 1 & 0.5536 & 0.5547 & 0.5543 & 0.5540 & 0.5543 \\
Random 2 & 0.5567 & 0.5546 & 0.5546 & 0.5547 & 0.5544 \\
Random 3 & 0.5547 & 0.5545 & 0.5546 & 0.5547 & 0.5547 \\
\hline
\end{tabular}

Table 3: Influence of random distributions of fibers on the effective thermal conductivity of composites with $v_{f}=54 \%$

\begin{tabular}{lccccc}
\hline & \multicolumn{5}{c}{ Number of fibers } \\
& 50 & 100 & 200 & 400 & 600 \\
\hline Random 1 & 0.6849 & 0.6854 & 0.6841 & 0.6853 & 0.6855 \\
Random 2 & 0.6823 & 0.6875 & 0.6884 & 0.6861 & 0.6851 \\
Random 3 & 0.6861 & 0.6549 & 0.6861 & 0.6856 & 0.6862 \\
\hline
\end{tabular}

crease with the increase of test temperature, but increase with the increase of fiber volume fraction. Moreover, for each value of volume fraction, both the regular and random fiber packings give almost same predictions, which are always smaller than that from the theoretical model. Besides, it is found from Fig. 12 that for the 50 fiber model with $27 \%$ fiber volume fractions, there is a big difference to other predictions, while such difference becomes slight as the increase of the number of fibers. This can be attributed to the random arrangement of fibers in the unit cell.

\subsection{The influence of fiber volume fraction}

Fig. 13 compares the effective thermal conductivity of composite containing randomly dispersed fibers retrieved numerically with that predicted by the regular models, the theoretical model and the experiment as a function of fiber volume fraction ranging from $5 \%$ to $65 \%$, which covers low, medium and high values of fiber volume fraction. The numbers of fibers in the composite unit cells are 50,100, 200,400 , respectively. The thermal conductivity of matrix material is taken as $0.4367 \mathrm{~W} /(\mathrm{mK})$, which corresponds to $65^{\circ} \mathrm{C}$ test temperature. Fig. 13 indicates that $k_{x}$ nonlinearly increases as $v_{f}$ increases for the given thermal properties of fiber and matrix materials. More importantly, the numerical predictions from the regular and random models are in better agreement with the experimental results, compared with the theoretical predictions. Besides, it is also resulting that the transverse thermal conductivity of the random array model is higher than those from regular array model.

\subsection{The influence of thermal property contrast}

To investigate the influence of the thermal conductivity mismatch between the fiber and matrix phases, Fig. 14 plots the effective thermal conductivity $k_{x}$ of a composite material containing regularly or randomly distributed fibers as a function of matrix thermal conductivity $k_{m}$ ranging from 0.1 to $1000 \mathrm{~W} /(\mathrm{mK})$ for the fiber volume fraction $v_{f}=50 \%$. The fiber thermal conductivity keeps $1.03 \mathrm{~W} /(\mathrm{mK})$ unchanged. It is demonstrated from Fig. 14 that $k_{x}$ looks to almost linearly increase as $k_{m}$ increases in the full range and the discrepancy between the theoretical and numerical predictions is expected to linearly increase as the thermal conductivity contrast increases. However, the results in Fig. 15 indicate that this phenomenon of linear increase is not true when the matrix thermal conductivity is obviously smaller than the fiber thermal conductiv- 

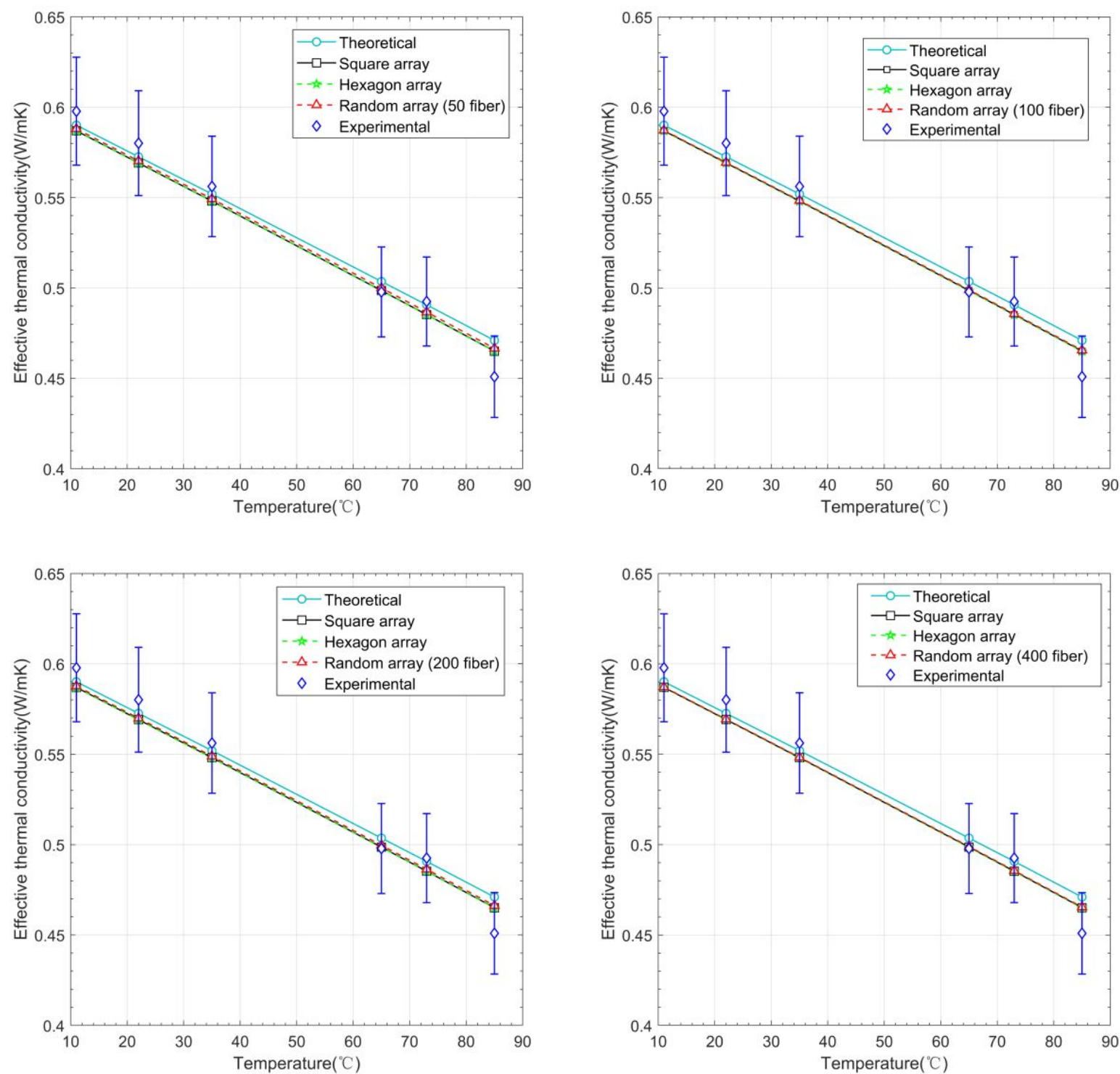

Figure 11: Effective thermal conductivity for different test temperatures with $v_{f}=14 \%$

ity. From Fig. 15, it is found that the effective thermal conductivity $k_{x}$ shows apparent nonlinear behavior when the matrix thermal conductivity changes from 0.1 to $2 \mathrm{~W} /(\mathrm{mK})$. Although the numerical models always give lower predictions than the theoretical model, they can give same results when $k_{m}=k_{f}$. This also demonstrates the validation of the computational tool. More importantly, the effective thermal conductivity from the regular models is smaller than that from the random permutation models when $k_{m}$ changes in the range of $[0.1,1.03] \mathrm{W} /(\mathrm{mK})$, while it becomes greater when the matrix thermal conductivity changes from 1.03 to $1000 \mathrm{~W} /(\mathrm{mK})$. Besides, as the material mismatch increases, the influence of the number of fibers becomes significant.

\subsection{The influence of fiber radius}

Another interesting issue is to investigate the effect of fiber radius on the effective thermal conductivity of composite. To do so, the thermal conductivity of the matrix and the fiber are assumed to be 0.4367 and $1.03 \mathrm{~W} /(\mathrm{mK})$, respectively. Fig. 16 displays the variation of effective thermal conductivity in terms of fiber radius for the volume fraction of $50 \%$ and the number of fibers is 100. From Fig. 16, 

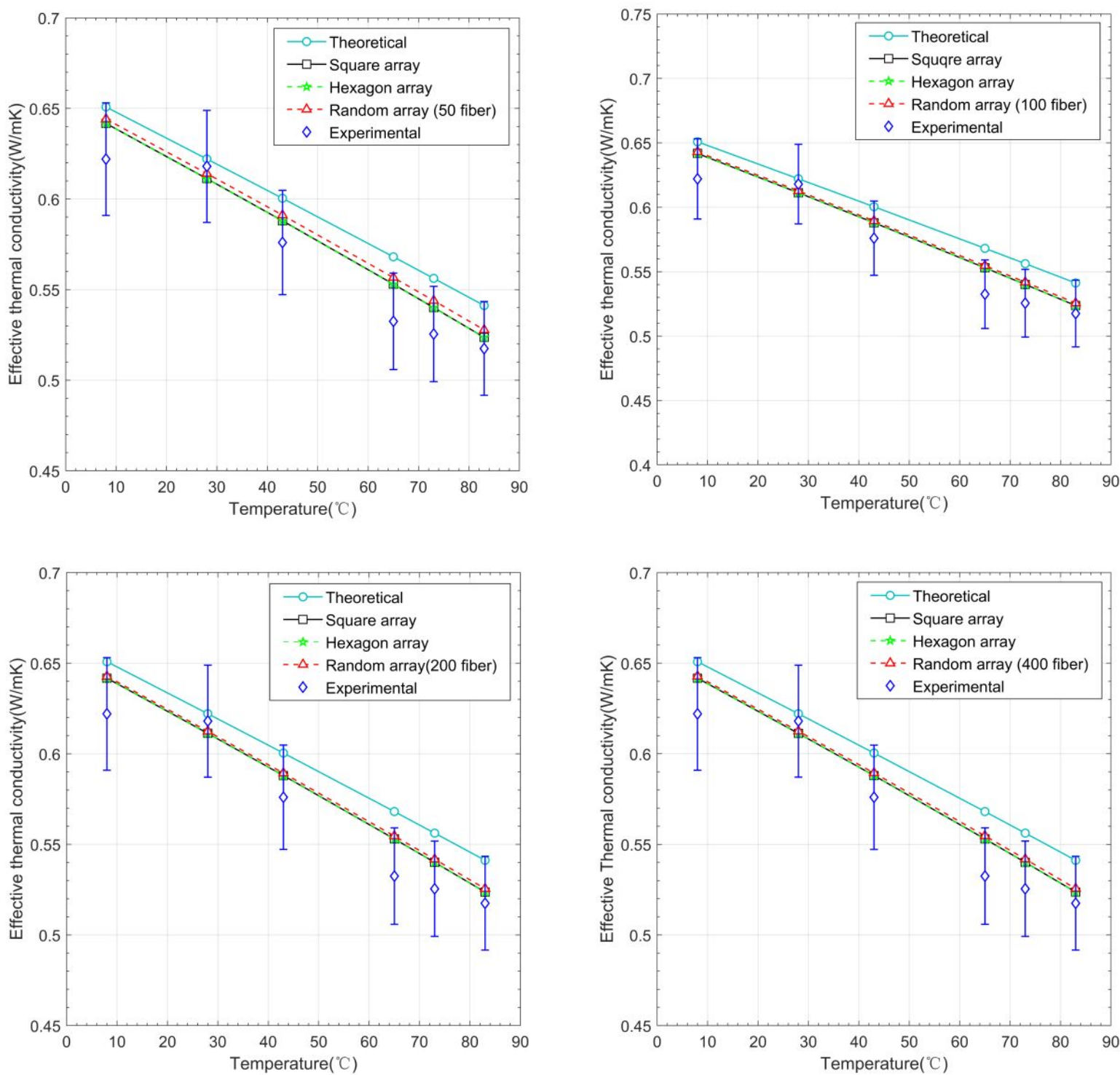

Figure 12: Effective thermal conductivity for different test temperatures with $v_{f}=27 \%$

it is found that the change of fiber radius has a little influence on the effective thermal conductivity of the random composite. Besides, for the special case that the fiber size is not uniform, as indicated in Fig. 17, the effective thermal conductivity of the composite with $50 \%$ fiber volume fraction is found to be $0.6614 \mathrm{~W} /(\mathrm{mK})$, which is slightly different to the case of uniform fiber size.

\section{Conclusions}

This study investigates the correlation of the effective transverse thermal conductivity of unidirectional fiber reinforced composite and the related geometric parameters, the thermal conductivity contrast and the test temperature. The predefined geometric parameters for the reconstruction of composite microstructure include the fiber random arrangement, the number of fibers and the fiber volume fraction. Results indicate that (1) Due to the dependence of matrix thermal conductivity on the test temperature, the effective thermal conductivity of composite is also 


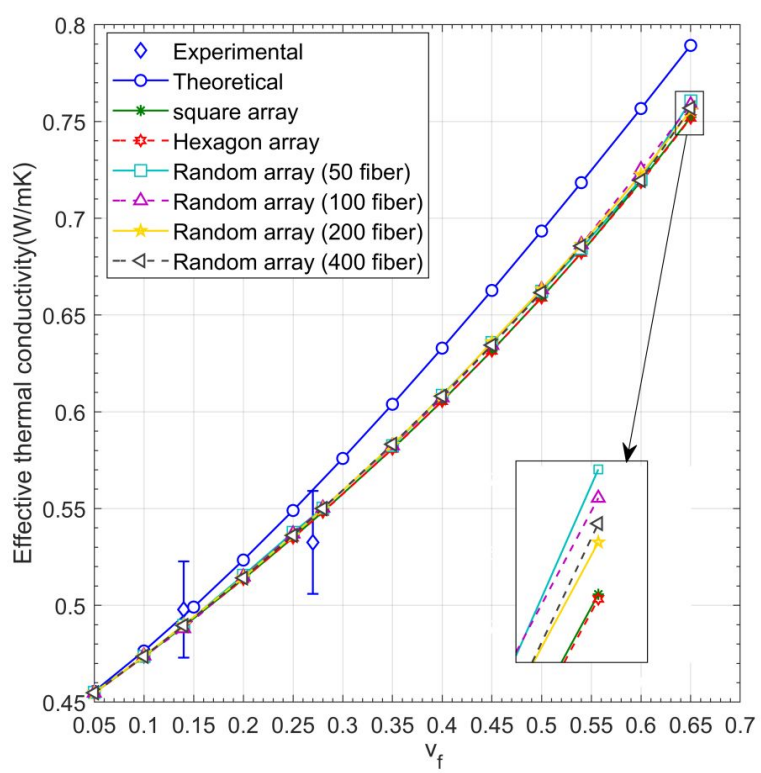

Figure 13: Effective thermal conductivity of composite in terms of fiber volume fraction

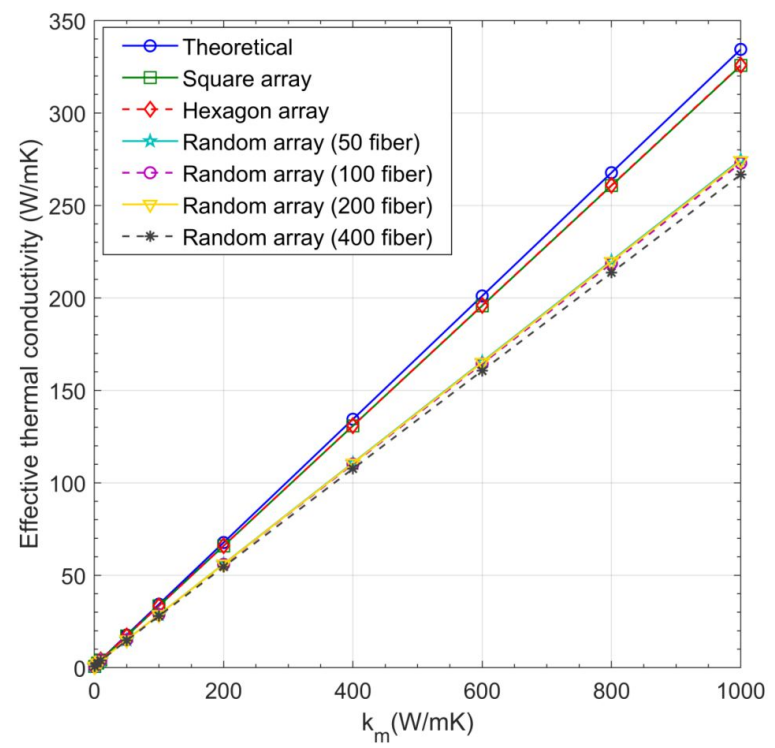

Figure 14: Effective thermal conductivity of composite in terms of the matrix thermal conductivity

expected to be a function of test temperature; (2) The effective thermal conductivity of composite from the random array model is in good agreement with the experimental prediction, but it is always lower than the theoretical prediction; (3) Compared to other geometric parameters, the fiber volume fraction plays a more important role to the effective thermal conductivity of composite. (4) The influence of fiber quantity becomes obvious as the thermal con-

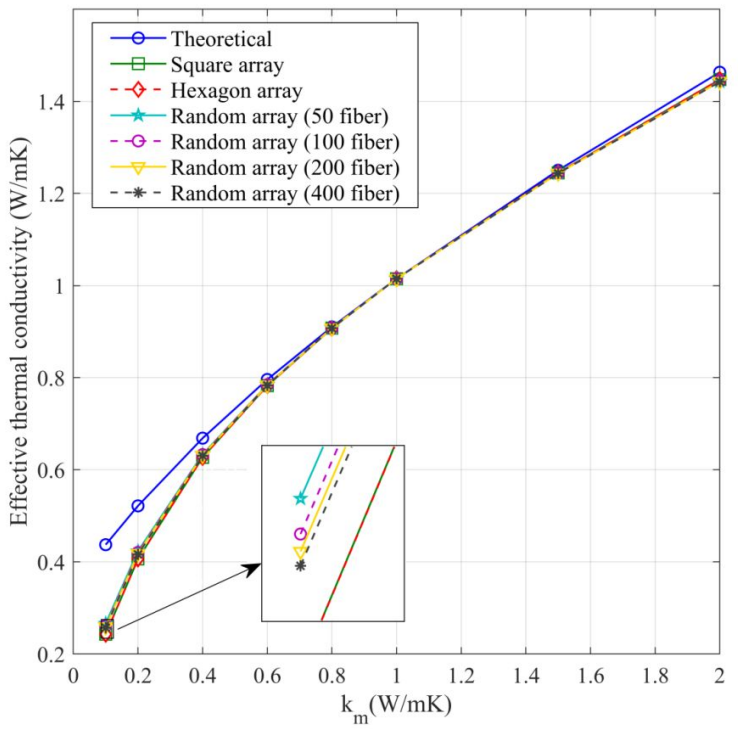

Figure 15: Effective thermal conductivity of composite in terms of the matrix thermal conductivity

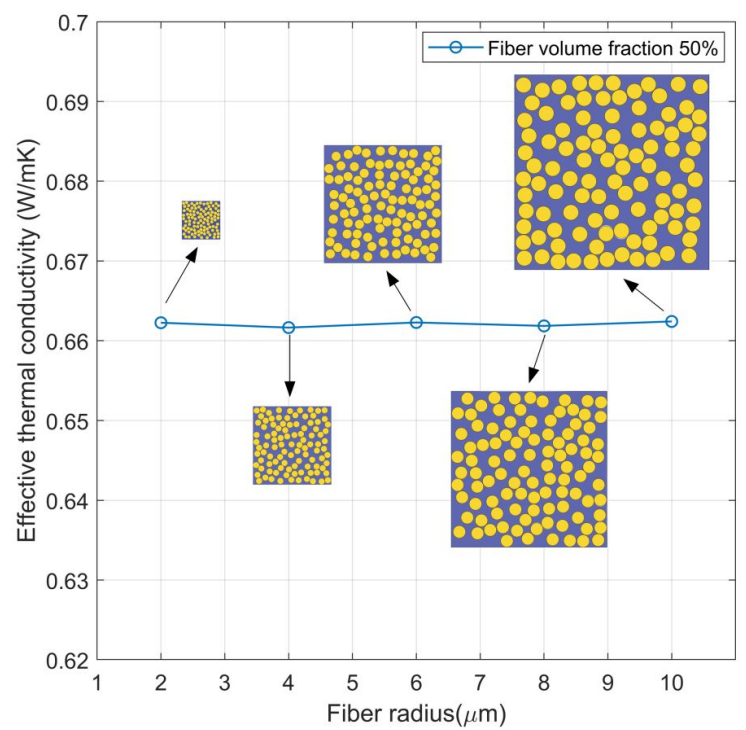

Figure 16: Effective thermal conductivity of composite in terms of the fiber radius

ductivity mismatch between the fiber and matrix phases dramatically increases. (5) The effective thermal conductivity of composite changes linearly when the matrix thermal conductivity is greater than the fiber thermal conductivity, while it nonlinearly varies when the matrix thermal conductivity is smaller than the fiber thermal conductivity. (6) The change of fiber size has a little influence to the effective thermal conductivity of random composite. 


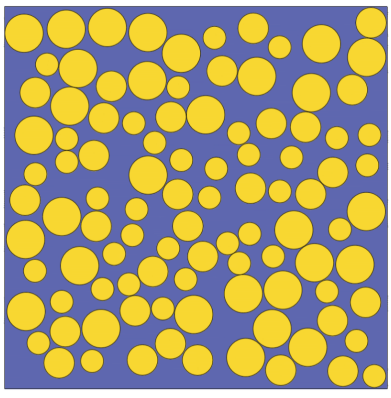

Figure 17: The generated random model with nonuniform fiber size for the case of 100 fibers and $v_{\mathrm{f}}=50 \%$

Acknowledgements: The work described in this paper was partially supported by the Program for Innovative Research Team of Science \& Technology of Henan Province of China (Grant no. 19IRTSTHN020) and Key Project of Henan Educational Committee (Grant no. 13A560169).

\section{References}

[1] Arasu AV, Sornakumar T, Sol. Energy 2007, 81, 1273-1279.

[2] Beckermann GW, Pickering KL, Compos. Part A-Appl 2008, 39, 979-988.

[3] Bahmani A, Li G, Willett TL, Montesano J, Compos. Struct. 2018, 192, 153-164.

[4] Makaremi M, Pasbakhsh P, Cavallaro G, Lazzara G, Aw YK, Lee SM, Milioto S, ACS Appl Mater Interfaces 2017, 9, 17476-17488.

[5] Bertolino V, Cavallaro G, Lazzara G, Milioto S, Parisi F, New J. Chem. 2018, 42, 8384-8390.

[6] Lazzara G, Cavallaro G, Panchal A, Fakhrullin R, Stavitskaya A, Vinokurov V, Lvov Y, Curr. Opin. Colloid Interface Sci. 2018, 35, 42-50.

[7] Rocha RPA, Cruz MAE, Numer. Heat Trans. Part A-Appl 2001, 39, 179-203.

[8] Xiao J, Xu Y, Zhang F, Compos. Struct. 2018, 189.

[9] Liu K, Takagi H, Osugi R, Yang Z, Compos. Sci. Technol. 2012, 72, 633-639.

[10] Villière M, Lecointe D, Sobotka V, Key Eng. Mater. 2013, 504506, 1091-1096.
[11] Koráb J, Štefánik P, Kavecký Š, Šebo P, Korb G, Compos. Part AAppl 2002, 33, 577-581.

[12] Wang M, Kang Q, Pan N, Appl. Therm. Eng. 2009, 29, 418-421.

[13] Hasselman DPH, Donaldson KY, Thomas JR, J. Compos. Mater. 1993, 27, 637-644.

[14] Markworth AJ, J. Mater. Sci. Lett. 1993, 12, 1487-1489.

[15] Wang H, Xiao Y, Qin QH, Sci. Iran. 2016, 23, 268-276.

[16] Wang H, Zhao XJ, Wang JS, Compos. Sci. Technol. 2015, 118, 117126.

[17] Sun CT, Vaidya RS, Compos. Sci. Technol. 1996, 56, 171-179.

[18] Gusev AA, Hine PJ, Ward IM, Compos. Sci. Technol. 2000, 60, 535-541.

[19] Huang Y, Jin KK, Ha SK, J. Compos. Mater. 2008, 42, 1851-1871.

[20] Jin KK, Oh JH, Ha SK, 2007, 41, 591-611.

[21] Lei YP, Wang H, Qin QH, Sci. Eng. Compos. Mater. 2018, 25, 143.

[22] Beicha D, Kanit T, Brunet Y, Imad A, Moumen AE, Khelfaoui Y, Mech. Mater. 2016, 102, 47-53.

[23] Bouaoune L, Brunet Y, El Moumen A, Kanit T, Mazouz H, Composites Part B 2016, 103, 68-73.

[24] Dong L, Atluri SN, Comput. Mater. Continua. 2012, 30, 39-81.

[25] Dong L, Atluri SN, Comput. Model. Eng. Sci. 2012, 83, 183-219.

[26] Dong L, Atluri SN, Comput. Mater. Continua. 2012, 29, 169-211.

[27] Zhang B, Yang Z, Sun X, Tang Z, Comp. Mater. Sci. 2010, 49, 645651.

[28] Garcea SC, Wang Y, Withers PJ, Compos. Sci. Technol. 2018, 156, 305-319.

[29] Wang H, Lei YP, Wang JS, Qin QH, Xiao Y, J. Compos. Mater. 2016, 50, 1509-1521.

[30] Fang Z, Li M, Wang S, Li Y, Wang X, Gu Y, Liu Q, Tian J, Zhang Z, Appl. Compos. Mater. 2017, 1-14.

[31] Yang L, Yan Y, Ran Z, Liu Y, Compos. Sci. Technol. 2013, 76, 1420.

[32] Li G, Sharifpour F, Bahmani A, Montesano J, Mater. Design 2018, 150, 124-138.

[33] Pathan MV, Tagarielli VL, Patsias S, Baiz-Villafranca PM, Compos. Part B-Eng 2017, 110, 267-278.

[34] Tavman IH, Akinci H, Int. Commun. Heat Mass Trans. 2000, 27, 253-261.

[35] Dai YJ, Tang YQ, Fang WZ, Zhang H, Tao WQ, Appl. Therm. Eng. 2018, 128, 1634-1645.

[36] Holman JP, McGraw-Hill, 1992.

[37] Springer GS, Tsai SW, J. Compos. Mater. 1967, 1, 166-173. 